\title{
Does glaucoma medication influence the diameter of the retinal arteriole in the human eye? (A pilot study using the Retinal Vessel Analyser)*
}

\begin{abstract}
P. Kóthy, G. Holló
1st Department of Ophthalmology, Semmelweis University, Budapest, Hungary

Received: December 13, 2001

Accepted: March 5, 2002

Purpose: To investigate the potential in vivo influence of different topical glaucoma medications on the diameter of the retinal arterioles of healthy volunteers and glaucoma patients.

Methods: The diameter of one pre-selected retinal arteriole per eye was measured using the Retinal Vessel Analyser (RVA), an instrument developed for non-invasive clinical measurement of the diameter of the main retinal vessels. The instrument contains a video system, and the integrated software recognises the boundaries of the retinal vessels by detecting their light-transmission profile. The vessel diameter (in arbitrary units) is plotted against time (seconds) on a separate display screen. In Study I the vessel diameter was measured in 12 eyes of six healthy volunteers (age 21-26 years, mean age 24.0 years) on six occasions each separated by 14 days. In a double-masked fashion, each subject's right eye was treated with one of 5 glaucoma medications (brinzolamide $1 \%$, timolol $0.5 \%$, betaxolol $0.5 \%$, brimonidine $0.2 \%$ or latanoprost $0.005 \%$ ) and the left eye always received balanced salt solution. In Study II, one randomly selected eye of 16 patients (age 50-79 years, mean age 65.2 years) suffering from primary openangle glaucoma controlled with topical monotherapy was investigated, in an unmasked fashion. Four patients were on betaxolol $0.5 \%$ treatment, six subjects were receiving non-selective topical beta receptor blockers and six subjects were being treated with once daily latanoprost $0.005 \%$.
\end{abstract}

Correspondence should be addressed to

Péter Kóthy, M.D.

1st Department of Ophthalmology

Semmelweis University

H-1083 Budapest, Tömő u. 25-29, Hungary

Fax: +36-1-210-0309

E-mail: kotpet@szem1.sote.hu

* Supported by a Hungarian national grant for medical research, ref. no: ETT 293/2000. The authors have no financial interest in any drug or instrument used in the study. 
Results: The coefficient of variation for the arteriole diameter in the healthy volunteers was less than $12 \%$ in each case. No significant post-treatment change of the diameter of the preselected arteriole was found for any topical medication investigated, either in the healthy volunteers (Study I) or in the patients suffering from glaucoma (Study II) ( $>0.05$, paired $t$-test). In addition, in Study $I$ no difference was observed in the alteration of the arteriole diameter between the baseline and the hour 2 measurements when the values from the drug-treated and placebo treated eyes were compared ( $p>0.05$, two-way ANOVA).

Conclusion: In the present investigations it was not possible to detect any statistically meaningful change of the arteriole diameter at two hours after the instillation of any of several topical antiglaucoma drugs widely used in clinical practice. Further investigations are necessary to clarify whether the lack of observed change is due to the lack of retinal vascular effects of the drugs investigated, or is due to an inability of the RVA instrument in practice to detect alterations between time-points separated by several hours.

Keywords: glaucoma, retinal vessel analyser, retinal arterioles, topical glaucoma medication

In recent years an increasing amount of evidence has been found demonstrating impairment of the ocular blood flow in glaucoma (9). Though the potential role of pathological vasoregulation in the development of glaucoma is strongly supported by in vitro studies $(5,16,33)$ and in vivo animal models $(2)$, the clinical detection of ocular blood flow impairment is still difficult, and the results are contradictory. Since some of the topical medications used in clinical practice to decrease intraocular pressure (IOP) in glaucoma have been shown to also exert vasorelaxing properties in experimental models of glaucoma $(2,5,16,34)$ it would be of great clinical significance if such effects in the glaucomatous eye could be verified or disproved. However, measuring the fine changes of the ocular circulation in the clinical context in a non-invasive manner, and correlating the measurements with the potential vascular effects of the topical medication, is a technically difficult problem. Using colour Doppler imaging available today, blood flow velocity can be measured and resistive indices calculated for the retrobulbar vessels and for the central retinal artery $(1,32)$. However these vessels provide the arterial blood supply for the whole eye, and so colour Doppler imaging is not specific enough to be used for characterising the optic nerve head or retinal blood supply separately; and unfortunately this technique cannot image small-diameter vessels. Similarly, measurement of the pulsatile component of the ocular blood flow provides information regarding the blood supply to the whole eye, and seems to show a wide overlap between the values for healthy eyes and for eyes suffering from glaucoma $(10,20)$. Using laser Doppler flowmetry $(20,27)$ the blood flow can be measured in a small segment of the neuroretinal rim and the retina; but the measurement depth is not well-defined, which means that some choroidal blood-flow is probably also measured. 
However, choroidal blood flow represents approximately $85 \%$ of the total ocular blood flow; and moreover since the vasoregulation in the retina and the choroid are different (32), even a small effect from this layer might considerably influence the retinal blood flow measurements, and thus mask the potential drug-related alterations. The results with scanning laser Doppler flowmetry, which provides information on capillary perfusion in the retina and optic nerve head, are contradictory, and the variability of the measurements is high $(14,18)$.

The Retinal Vessel Analyser (RVA; IMEDOS GmbH, Weimar, Germany; Figs 1 and 2) is a recently-developed instrument for non-invasive clinical measurement of the diameter of the major retinal arterioles and veins $(3,4,28)$. The RVA provides the possibility to investigate the major retinal arteries or veins individually, which allows investigation of a vascular area different from the ones measured by any of the other non-invasive clinical techniques used to study the ocular blood flow. The technique is shown to be appropriate for the recognition of hyperacute alterations of the diameter, e.g. in studies using $\mathrm{O}_{2}$ or $\mathrm{CO}_{2}$ inhalation $(21,31)$, systemic vasoconstrictors (28) or vasodilators, or photostimulation of the retina $(11,29)$. However to our knowledge few investigations have been reported on the use of the RVA for the evaluation of the potential vascular effects of topical glaucoma medication (24). In this study we investigated whether the RVA instrument can be used for the detection of vascular alterations in the retina caused by topical drugs used to lower the IOP.

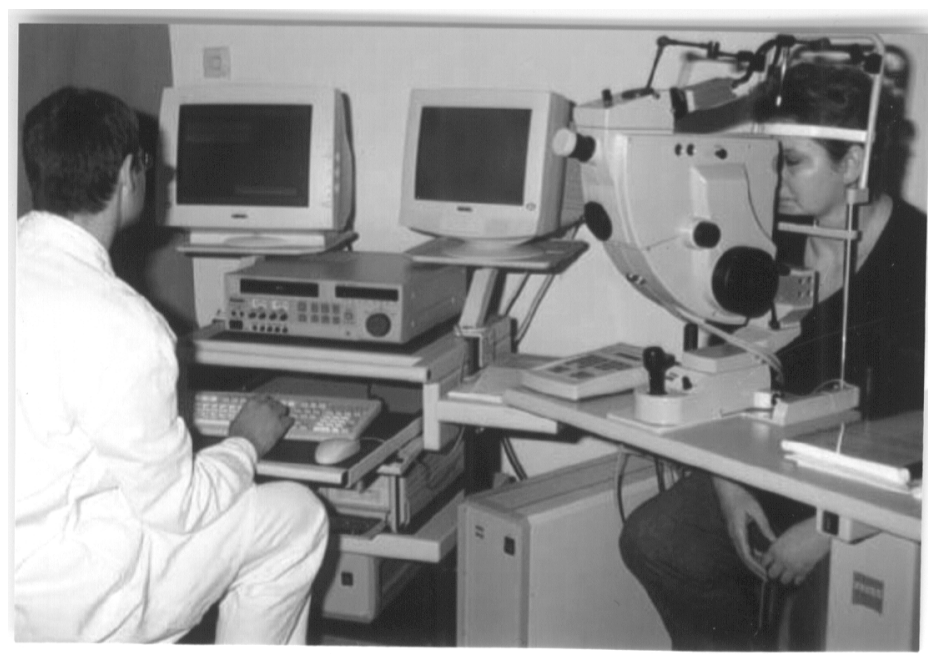

Fig. 1. Examination with the Retinal Vessel Analyser 


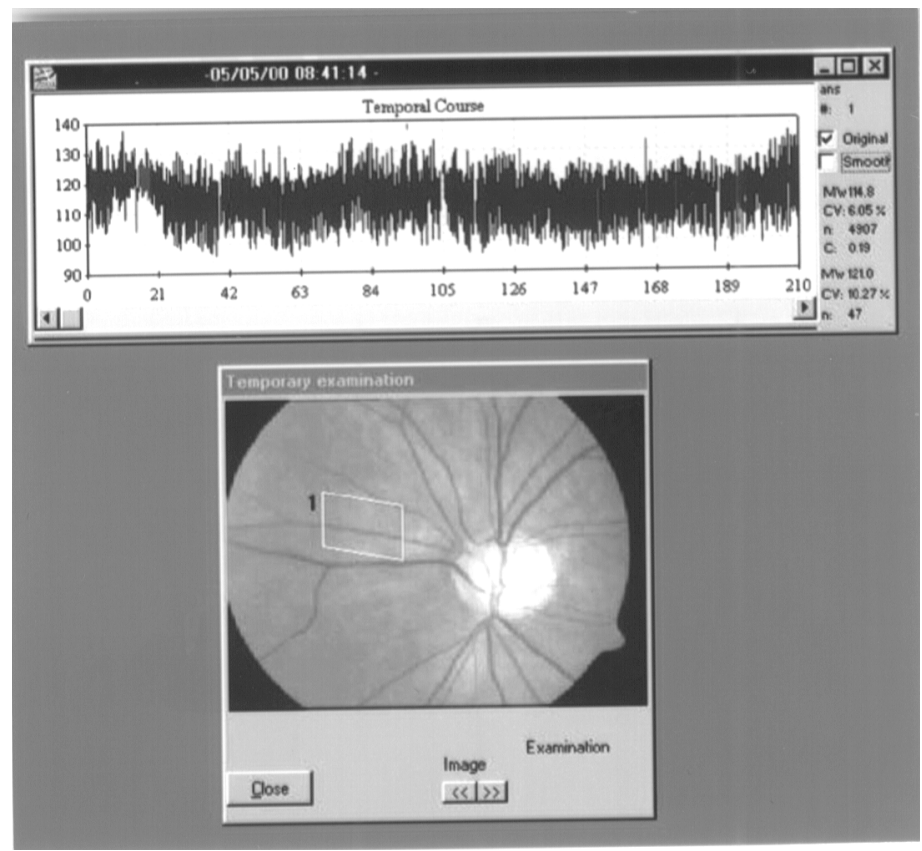

Fig. 2. Image of a pre-selected retinal arteriole of approximately $90 \mu \mathrm{m}$ within the frame area, and the graph of its measured diameter (arbitrary units) plotted against time (seconds)

\section{Subjects and Methods}

The Retinal Vessel Analyser (RVA; IMEDOS GmbH, Weimar, Germany) is a commercially available system which contains a fundus camera (Zeiss FF 450, Carl Zeiss GmbH, Jena, Germany), a digital video camera, a real-time video display screen, and a personal computer with analysis software which can determe the diameter of the imaged blood vessel, where this is greater than approximately $60 \mu \mathrm{m}$. The measurement principle is based on the recognition of the specific light-transmittance profile of the haemoglobin-containing blood within the vessel. Measurement of retinal vessel diameters is based on adaptive algorithms using individual vessel profiles. When a specific vessel profile is recognised the RVA system is able to follow this vessel along as it appears within the measurement frame (28). To select a region of interest the investigator defines a rectangle enclosing the relevant part of the desired vessel on the image on the real-time display screen. After this the measurement of vessel diameters can be started. Vessel diameter is calculated along the segment of vessel which lies within the frame. The system can automatically correct for eye movements, provided the 
vessel is still within the frame. The vessel diameter defined in relative units can be recorded as a function of time as well as a function of the position along the axis of the vessel. According to the manufacturer (IMEDOS GmbH: The Retinal Vessel Analyser, 1997) 1 unit of the vessel diameter as measured with the RVA corresponds to $1 \mu \mathrm{m}$ vessel diameter in the Gullstrand's eye model. However, since the optical properties of the individual eyes show a significant variation, this relation can be considered only an approximation in real clinical use of the instrument. Retinal vessel diameters are determined in real time with a maximum frequency of 25 readings per second. The fundus image can be observed on the real-time screen, and if necessary stored on a video recorder. Evaluation of the retinal vessel diameters can be done on-line, or offline from the recorded video tape.

The clinical investigation protocol was approved by the Ethics Committee of the University and written informed consent was obtained from all participants. In Study I, 12 eyes of six healthy volunteers (age 21 to 26 years, mean age 24.0 years) were investigated in a double masked, placebo controlled fashion. Each volunteer was a non smoker and was healthy, was using no systemic or ocular medication at the time of the enrolment, and had a negative ocular medical history. Each subject underwent a detailed ophthalmological examination before enrolment. The evaluation consisted of visual acuity measurement, visual field testing with the Octopus perimeter Dynamic G2 test, IOP measurements with Goldmann tonometry, slit-lamp examination, and funduscopy with dilated pupils. The visual functions were normal, and the optic nerve heads showed no pathological alterations as examined using a 90 diopter non-contact aspherical lens and a slit lamp for stereoscopic viewing. The IOP was lower than $22 \mathrm{~mm} \mathrm{Hg}$ in all cases.

The RVA measurements were performed on both eyes in the morning hours on six occasions separated by intervals of 14 days, by the same examiner (P.K.). Pupils were dilated with a drop of tropicamide $\mathrm{HCl} 0.5 \%$ (Mydrum, Chauvin Ankerpharm GmbH, Rudolstadt, Germany). The baseline RVA, blood pressure and pulse rate measurements were performed with the subject in a sitting position, following 10 minutes rest in the same position. Then one of the topical glaucoma drugs; brinzolamide 1\% (Azopt, Alcon Inc., Fort Worth, TX, USA), timolol 0.5\% (Cusimolol, Alcon Cusí S.A., El Masnou, Spain), betaxolol 0.5\% (Betoptic, Alcon-Couvreur Inc., Puurs, Belgium), brimonidine $0.2 \%$ (Alphagan, Allergan Inc., Irvine, CA, USA), latanoprost $0.005 \%$ (Xalatan, Pharmacia Corp., Peapack, NJ, USA) was instilled in the right eye and balanced salt solution in the left eye, in a double-masked fashion. The measurements were repeated two hours later, again after a 10-minute rest. The participants spent the two-hour interval resting, and took no medication or coffee. Before using any glaucoma drops, as a control experiment, on a separate occasion two 
RVA measurements separated by a time interval of 2 hours were performed through dilated pupils but using balanced salt solution in both eyes of all patients, in order to estimate the coefficient of variation of the RVA measurements.

In Study II, 16 patients (age 50-79 years, mean age 65.2 years) suffering from primary open-angle glaucoma controlled with topical monotherapy were investigated. Over a period of at least 3 months their IOP had been consistently lower than $22 \mathrm{~mm} \mathrm{Hg}$ while using a single topical medication. One randomly selected eye per subject was analysed. Four of these eyes received the beta-1 receptor selective blocker betaxolol $0.5 \%$ twice daily, six were treated with non-selective beta receptor blocker eye drops of $0.5 \%$ concentration twice daily, and six eyes were on a single evening dose of latanoprost $0.005 \%$. Systemic medication where used was stopped for 24 hours before the day of the RVA measurement. The patients instilled their usual topical medication on the evening before the day of the investigation (except for latanoprost-treated patients, whose dose was omitted on the preceding evening). The baseline RVA, blood pressure and heart rate measurements were performed at 8 o'clock in the morning, in a similar way to those described in Study I. Then the topical medication was instilled in an open fashion, and two hours later the measurements were repeated.

In both studies a temporal (superior or inferior) main arteriole was selected for the measurement. Data for the pre-selected vessel segments was recorded for at least a 100 -second time period, then the images were examined for quality. Only high quality images were stored and used for later analysis. Detailed analysis was performed after the measurements for both studies were completed, but before unmasking the details of treatment for participants in Study I. Vessel diameter graphs for the first 100 seconds of each measurement were used for the automatic calculation of the mean diameter, and these mean values were used for the further analysis.

Reproducibility was defined as intraindividual standard deviation divided by the overall mean value (coefficient of variation). The paired $t$-test with Bonferroni correction was used to investigate the changes of the vessel diameter, blood pressure and heart rate between the baseline and the hour 2 measurements for each different medication, respectively. For Study I, two-way ANOVA was used to compare the change of the vessel diameter between the eye receiving topical medication and the contralateral, placebo-treated eye for each type of topical medication. P-values less than 0.05 were considered as significant.

\section{Results}

The coefficient of variation for the arteriole diameter in the healthy volunteers was less than $12 \%$. The alteration of the arteriole diameters for Study I are shown in Table I. 


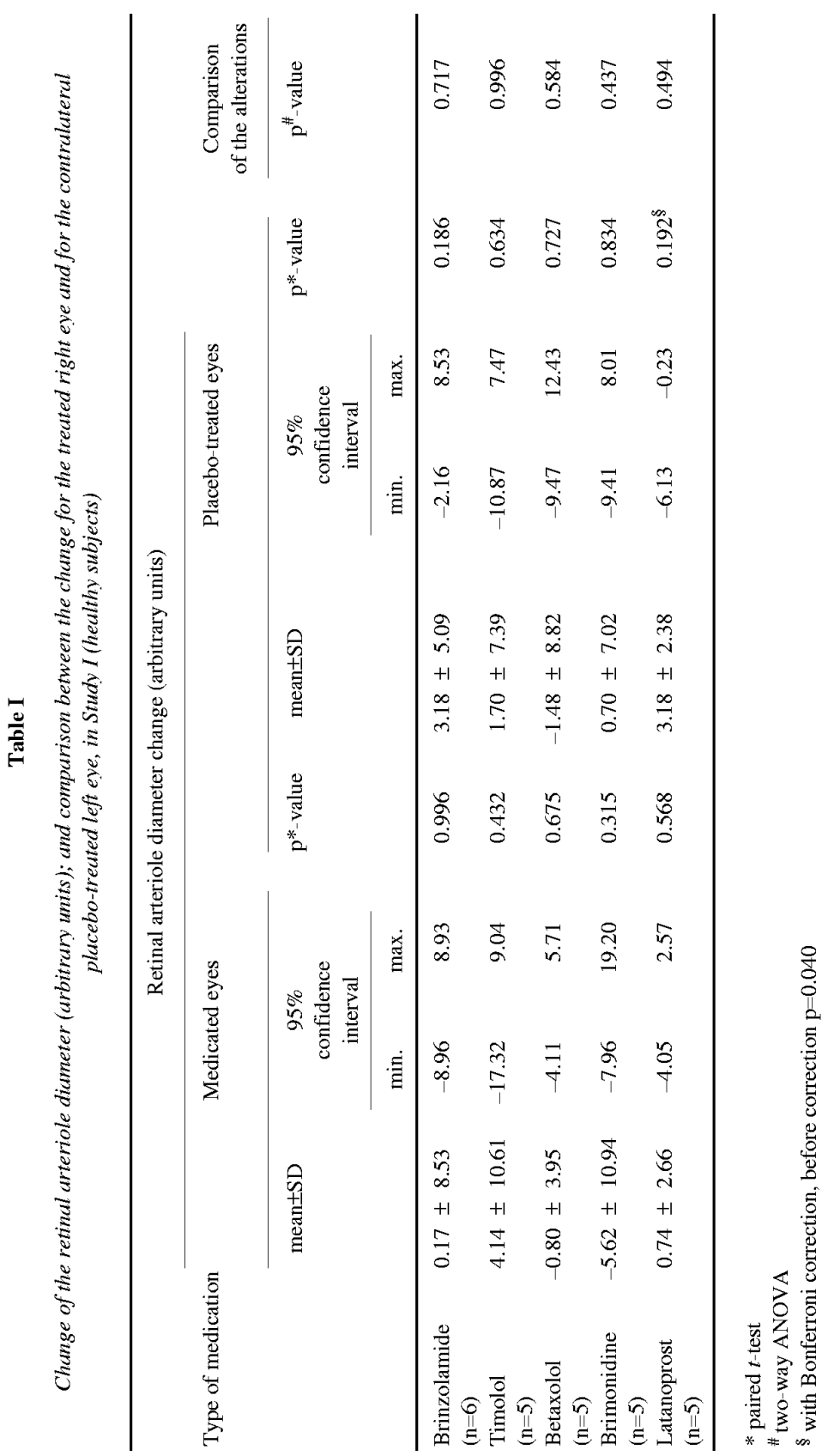




\section{Table II}

Change of the retinal arteriole diameter (arbitrary units) in Study II (glaucoma patients)

\begin{tabular}{|c|c|c|c|c|}
\hline \multirow{3}{*}{ Type of medication } & \multicolumn{3}{|c|}{$\begin{array}{l}\text { Retinal arteriole diameter change } \\
\text { (arbitrary units) }\end{array}$} & \multirow{3}{*}{$\mathrm{p}^{*}$-value } \\
\hline & \multirow[t]{2}{*}{ mean $\pm \mathrm{SD}$} & \multicolumn{2}{|c|}{$\begin{array}{l}95 \% \text { confidence } \\
\text { interval }\end{array}$} & \\
\hline & & $\min$. & $\max$. & \\
\hline Non-selective $\beta$-receptor blockers $(n=6)$ & $0.033 \pm 3.120$ & -3.241 & 3.308 & 0.980 \\
\hline Betaxolol $0.5 \%(n=4)$ & $-0.050 \pm 4.183$ & -6.706 & 6.606 & 0.982 \\
\hline Latanoprost $0.005 \%(\mathrm{n}=6)$ & $-1.283 \pm 4.739$ & -6.256 & 3.689 & 0.536 \\
\hline
\end{tabular}

* paired $t$-test

No significant change was found for eyes receiving any type of topical medication, or for the placebo treatment; except for a single change in the placebo-treated left eyes on the day of the latanoprost treatment (Table I). This apparent difference, however, lost its significance after Bonferroni correction (corrected $\mathrm{p}=0.192$ ). When the change of the arteriole diameter was compared between the medication-treated eye and the placebotreated eye for each session, again no difference was seen (Table I). The systolic and the diastolic blood pressure as well as the heart rate showed no difference between the baseline measurement and the hour 2 examination ( $p>0.05$ in all cases, detailed data not shown).

The alterations of the retinal arteriole diameter for the glaucoma patients in Study II are shown in Table II. None of the diameter changes for any type of medication was significant. The systolic and the diastolic blood pressure, and the heart rate, remained unchanged between the baseline and the hour 2 measurements for each type of glaucoma medication ( $p>0.05$ in all cases, paired t-test; detailed data not shown).

\section{Discussion}

Some of the topical medications used to reduce IOP in glaucoma are reported to produce vasodilation of the precontracted vessels in vitro, and also in vivo in animal models of glaucoma $(2,5,16,34)$. However, such effects have not been consistently verified in human glaucoma patients. One of the reasons for the lack of clear evidence for the presence or absence of human retinal vasoactivity exerted by topical medication is the lack of a clear understanding of the biological meaning of the results obtained with different types of non-invasive measurement techniques. Colour Doppler imaging and pulsatile ocular blood flow measurement provide information only for the total 
blood supply of the eye (32). Laser Doppler flowmetry and scanning laser Doppler flowmetry are not completely selective for the retina, and for the latter method the clinical results are conflicting $(14,18,20,27)$.

In view of these difficulties the Retinal Vessel Analyser, an instrument developed recently for the automatic measurement of the diameter of the main retinal vessels, in principle provides a possibility for a new, non-invasive, clinical approach to the problem. Since with the RVA it is not blood flow but vessel diameter which is measured directly, the interpretation of the findings would seem to be more straight-forward as compared to the above-mentioned techniques. The ability of the RVA to detect hyperacute vasoconstriction and relaxation has been verified in human experiments using systemic medication or $\mathrm{CO}_{2}$ and $\mathrm{O}_{2}$ inhalation $(17,21,25,28)$. In contrast to these studies in the present study we investigated whether the RVA is a suitable technique for the detection of vasoconstriction or vasodilation induced by topical IOPlowering medication.

All the eye drops investigated in our study are world-wide used topical drugs which are approved for lowering the intraocular pressure in glaucoma. In addition to that, there is indirect evidence for retinal vasoactivity exerted by these molecules. Betaxolol has been shown to relax endothelin-1 preconstricted isolated human arterioles in a dose dependent manner in vitro (16). This effect is mediated by L-type calcium channels and considered unrelated to the $\beta$-receptor activity of betaxolol $(23,34)$. Till now betaxolol is the only glaucoma medication which was systematically evaluated for drug concentration in the different ocular tissues including the retina in in vivo animal models and in human glaucoma patients $(8,15)$. The data from chronic medication studies show that both in animal models and in humans the retinal concentration of betaxolol is magnitudes higher than the minimum concentration necessary for in vitro vasorelaxation of the isolated human retinal arterioles $(8,15)$. These data, however, provide no information on the pharmacologically active ocular drug concentrations. Timolol, a non-selective $\beta$-receptor blocker with a significantly lower calcium channel blocking activity was also demonstrated to relax precontracted isolated mammalian arterioles in vitro, however, this effect was significantly weaker and required significantly higher concentration than that of betaxolol (16). Topical carbonic anhydrase medication improved blood flow in the ophthalmic artery, ciliary arteries and central retinal artery as measured with colour Doppler imaging, and was associated with an accelerated arterio-venous passage time in the retina both in humans and in animal models $(12,13,22)$. However, retinal and optic nerve head vasodilation was not investigated in these experiments. Since the accelerated retinal perfusion is always associated with a decrease of intraocular pressure in such studies, it is impossible to exclude the causative role of intraocular pressure reduction and the resulting increase of ocular perfusion pressure in the acceleration of the retinal perfusion (2). In contrast to 
the $\beta$-receptor blockers and the topical carbonic anhydrase inhibitors latanoprost, a prostaglandin $\mathrm{F}_{2 \alpha}$ analogue, and brimonidine, a relatively selective $\alpha 2$-receptor agonist are retinal vasoconstrictors in vitro $(6,7)$. On the other hand in in vivo models the retinal concentration of brimonidine was much below the value necessary to the stimulation of the $\alpha 1$-receptors, which are responsible for vasoconstriction $(7,19)$ and the presumed retinal concentration of topically applied latanoprost is also considerably lower than that used in the in vitro experiments.

Drug induced alterations of the human retinal and optic nerve head perfusion is extremely difficult to measure and interpret. The difficulties are caused by the anatomy of the primate optic nerve head and retina, the technical limitations of the different noninvasive measurement techniques and the several unknown characteristics of ocular drug delivery in and around the eye. In humans it is not possible to use invasive methods to evaluate pharmacokinetics and tissue specific activity of the topically applied ocular drugs. Therefore new and non-invasive measuring techniques are very important to be tried in real clinical conditions. Our study with the Retinal Vessel Analyser was designed for a typical clinical situation, and were investigated the potential effects of widely used topical glaucoma drugs on the major retinal arterioles. In contrast to some in vitro experiments, but similar to several other non-invasive clinical investigations $(14,26,30)$, we were unable to show any change between the baseline and the hour 2 post-medication readings either in healthy, young volunteers or in glaucoma patients under chronic topical monotherapy.

There are four possible explanations for our negative results. First, some hours are required for the development of any retinal vascular effect of a topically applied drug, since the molecule needs to penetrate from the ocular surface to the posterior layers of the eye (8). Such an interval, however, is too long for continuous recording with the RVA; therefore we had to pre-select a time interval between two separate measurements. The two-hour interval used in this study corresponds to the time of the maximum IOP-lowering effect of the majority of the topical glaucoma medications, but it is not clear whether the potential retinal effects have a similar time-profile to the IOP effect, in the human eye. This means that we might have missed the optimal time for the detection of the changes. The second explanation is based on the physiological alterations of the arteriole diameter in the retina (3). The natural alterations of the vessel diameter over time might have influenced the outcome of our investigations, by masking the drug-induced changes; even though the reproducibility of the RVA measurements for a 2 -hour time period was better than $12 \%$, for our healthy volunteers. The third possibility is the lack of any retinal effect of any of the topical drugs investigated, at the concentrations used. This however does not seem likely, since at least for the topical carbonic anhydrase inhibitors an acceleration of the retinal circulation has been reported two hours after the instillation of these drugs in the human eye $(12,22)$. The fourth 
possible explanation is that the vascular structure which is influenced by the drugrelated vasoconstriction or relaxation is distal from the main retinal arterioles, i.e. the small arterioles and the precapillaries, which unfortunately are too small to be investigated with the RVA.

The results of our pilot study suggest that the potential role of the Retinal Vessel Analyser in the detection of the possible vasoconstriction or vasodilation induced by topical glaucoma medication needs further investigation. In such studies, several different time-intervals should be used to compare the diameter of the retinal arteriole between the pre-treatment baseline and the post-treatment sessions.

\section{REFERENCES}

1. Altan-Yaycioglu R, Türker G, Acunas G, Izgi B: The effects of beta-blockers on ocular blood flow in patients with primary open angle glaucoma: a color Doppler imaging study. Eur. J. Ophthalmol. 11, 37-46 (2001)

2. Barnes G, Li B, Dean T, Chandler M: Increased optic nerve head blood flow after 1 week of twice daily topical brinzolamide treatment in Dutch-belted rabbits. Surv. Opthalmol. 44(S21), S131-140 (2000)

3. Blum M, Bachmann K, Wintzer D, Riemer T, Vilser W, Strobel J: Non-invasive measurement of the Bayliss effect in retinal autoregulation. Graefe's Arch. Clin. Exp. Ophthalmol. 237, 296-300 (1999)

4. Blum M, Bachmann K, Strobel J: Alterskorrelation der blutdruckinduzierten myogenen Autoregulation von menschlichen retinalen Arteriolen an 40 Probanden. Klin. Monatsbl. Augenheilkd. 217, 225-230 (2000)

5. Braakman R, van der Linden P, Sipkema P: Effects of topical $\beta$-Blockers on the diameter of the isolated porcine short posterior ciliary artery. Invest Ophthalmol. Vis. Sci. 40, 370-377 (1999)

6. Brogiolo G, Flammer J, Haefliger IO: Latanoprost is a vasoconstrictor in isolated porcine ciliary arteries. Klin. Monatsbl. Augenheilkd. 218, 373-375 (2001)

7. Cantor L, Burke J: Drug evaluation: brimonidine. Exp. Opin. Invest. Drugs 6, 1063-1083 (1997)

8. Dahlin D, Curtis M, DeSantis L, Struble C: Distribution of betaxolol to posterior ocular tissues of the cynomolgus monkey following a 30 days BID topical ocular regimen of betoptic S (ARVO abstract). Invest. Ophthalmol. Vis. Sci. 41(S4), S509 (2000)

9. Flammer J, Orgül S: Optic nerve blood-flow abnormalities in glaucoma. Progress in Retinal and Eye Research 17(2), 267-289 (1998)

10. Fontana L, Poinoosawmy D, Bunce C, O'Brien C, Hitchings R: Pulsatile ocular blood flow investigation in asymmetric normal tension glaucoma and normal subjects. Br J Ophthalmol. 82, 731-736 (1998)

11. Garhöfer G, Schmetterer L, Riva C: Strong constant light induces a decrease in the diameter of retinal vessels in light adapted healthy subjects (ARVO abstract). Invest. Ophthalmol. Vis. Sci. 42 (S4), S87 (2001)

12. Harris A, Arend O, Sung Chung H, Kagemann L, Cantor L, Martin B: A comparative study of betaxolol and dorzolamide effect on ocular circulation in normal-tension glaucoma patients. Ophthalmology 107, 430-434 (2000)

13. Harris A, Jonescu-Cuypers CP, Kageman L, Nowacki EA, Garzozi H, Cole G, Martin B: Effect of dorzolamide timolol combination versus timolol $0.5 \%$ on ocular blood flow in patients with primary open angle glaucoma Am. J. Ophthalmol. 132, 490-495 (2001) 
14. Holló G, van den Berg T, Greve E: Scanning laser Doppler flowmetry in glaucoma. International Ophthalmol. 20, 63-70 (1997)

15. Holló G, Whitson J, Faulkner RD, Wieland H, McCarty GR, Desantis L, Przydryga J, Dahlin DC: Betaxolol distributes to the retina, optic nerve head and choroid of the human eye following chronic topical administration of Betoptic S (abstract). Ophthalmic. Res. 33(S1), 153 (2001)

16. Hoste A: In vitro studies of the effects of $\beta$-adrenergic drugs on retinal and posterior ciliary microarteries. Surv. Ophthalmol. 43(S1), S183-190 (1999)

17. Jandrasits K, Luksch A, Söregi G, Dorner GT, Polak K, Schmetterer L: Effect of noradrenaline on retinal blood flow in healthy subjects. Ophthalmology 109, 291-295 (2002)

18. Kagemann L, Harris A, Chung H, Jonescu-Cuypers C, Zarfati D, Martin B: Photodetector sensitivity level and Heidelberg Retina Flowmeter measurements in humans. Invest. Ophthalmol. Vis. Sci. 42. 354-357 (2001)

19. Kent A, Nussdorf J, Fellows D, Small D, David R: Brimonidine concentrations in the posterior segment of patients during Alphagan treatment (abstract). J. Glaucoma 9, 117 (2000)

20. Kerr J, Nelson P, O'Brien C: A comparison of ocular blood flow in untreated primary open-angle glaucoma and ocular hypertension. Am. J. Ophthalmol. 126, 42-51 (1998)

21. Luksch A, Garhöfer G, Polak K, Schmetterer L: Effect of inhalation of different mixtures of $\mathrm{O}_{2}$ and $\mathrm{CO}_{2}$ on retinal blood flow (ARVO abstract). Invest. Ophthalmol. Vis. Sci. 42 (S4), S83 (2001)

22. Martinez A, Gonzalez F, Capeans C, Perez R, Sanchez-Salorio M: Dorzolamid effect on ocular bloodflow. Invest. Ophthalmol. Vis. Sci. 40, 1270-1275 (1999)

23. Melena J, Stanton D, Osborne NN: Comparative effects of antiglaucoma drugs on voltage-dependent calcium channels. Graefe's Arch. Clin. Exp. Ophthalmol. 239, 522-530 (2001)

24. Nagel E, Vilser W, Fuhrmann G, Lang GE: Einfluss des Topischen Carboanhydrasehemmers Dorzolamid (Trusopt) auf Retinale Astgefaessdurchmesser (abstract). Regulation und Quantifizierung der Okularen Perfusion IX. Workshop 1999, Hamburg, Germany, Abstract book 20 (1999)

25. Pache M, Meyer P, Prünte C, Orgül S, Nuttli I, Flammer J: Sildenafil induced retinal vasodilatation in healthy subjects. Br. J. Ophthalmol. 86, 156-158 (2002)

26. Pillunat LE, Böhm AG, Köller AU, Schmidt KG, Klemm M, Richard G: Effect of topical dorzolamide on optic nerve blood flow. Graefe's Arch. Clin. Exp. Ophthalmol. 237, 495-500 (1999)

27. Piltz-Seymour J, Grunwald J, Hariprasad S, DuPont J: Optic nerve blood flow is diminished in eyes of primary open-angle glaucoma suspects. Am. J. Ophthalmol. 132, 63-69 (2001)

28. Polak K, Dorner G, Kiss B, Polska E, Findl O, Rainer G, Eichler H, Schmetterer L: Evaluation of the Zeiss retinal vessel analyser. Br. J. Ophthalmol. 84, 1285-1290 (2000)

29. Polak K, Schmetterer L, Riva C: Influence of flicker frequency on flicker induced vasodilation (ARVO abstract). Invest. Ophthalmol. Vis. Sci. 41 (S4), S552 (2000)

30. Seong GJ, Lee HK, Hong YJ: Effects of $0.005 \%$ latanoprost on optic nerve head and peripapillary retinal blood flow. Ophthalmologia 213, 355-359 (1999)

31. Vilser W, Evans D, Braeuer-Burchardt C, Riemer T, Harris A, Lang GE, Lang GK: The validity of a new test to measure retinal vessel autoregulation response to $100 \%$ oxygen breathing (ARVO abstract). Invest. Ophthalmol. Vis. Sci. 39 (S4), S271 (1998)

32. Williamson T, Harris A: Ocular blood flow measurements. Br. J. Ophthalmol. 78, 939-945 (1994)

33. Wood J, DeSantis L, Chao H-M, Osborne N: Topically Applied Betaxolol Attenuates Ischaemiainduced Effects to the Rat Retina and Stimulates BDNF mRNA. Exp. Eye Res. 72, 79-86 (2001)

34. Yu DY, Su EN, Cringle S, Adler V, Yu P, DeSantis L: Systemic and ocular vascular roles of the antiglaucoma agents $\beta$-adrenergic antagonists and $\mathrm{Ca}^{++}$entry blockers. Surv. Ophthalmol. 43(S1), S214-222 (1999) 